\title{
THE IMPACT OF AGING AND HEARING STATUS ON VERBAL SHORT-TERM MEMORY
}

Clémence Verhaegen, Fabienne Collette, and Steve Majerus 


\section{INTRODUCTION}


$\square$ Cognitive decline in older adults

口 Short-term memory (e.g., Maylor, 1999)

$\square$ Decrease in hearing acuity

Age-related

hearing loss 
$\square$ Surprenant (2007):

$\square$ Adults $>70$ years

- 1/3 : clinically significant hearing loss

口 Almost $100 \%$ : mild hearing loss

$\square$ Standard STM tasks:

$\square$ span tasks $\rightarrow$ items presented auditorily 
$\square$ Baltes and Lindenberger (1997); Lindenberger and Baltes (1994):

$\square$ Cognitive functioning $\leftrightarrow$ Sensory functioning (vision and hearing)

- Especially old age

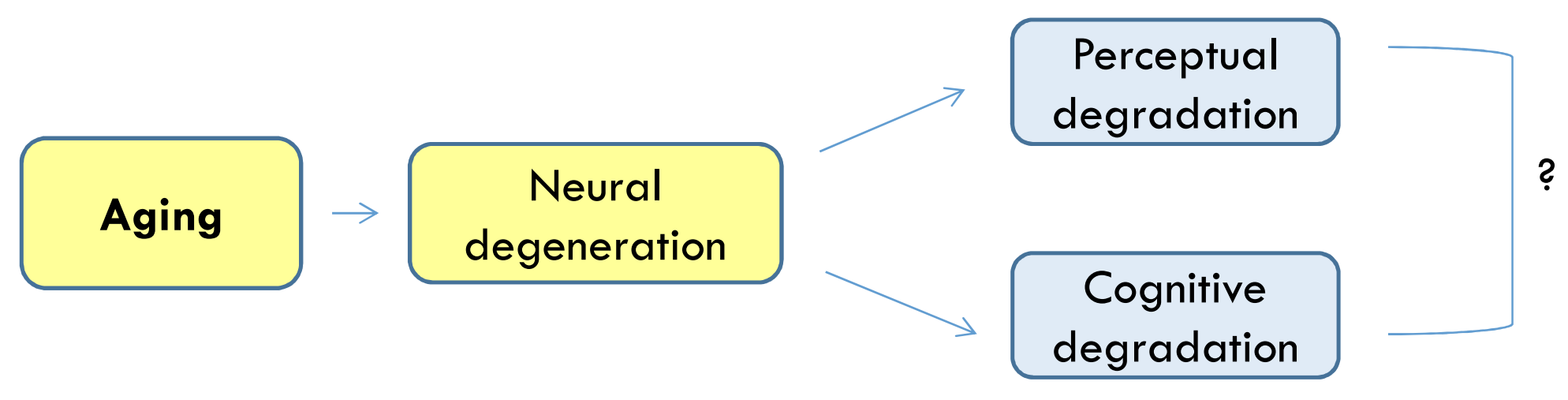




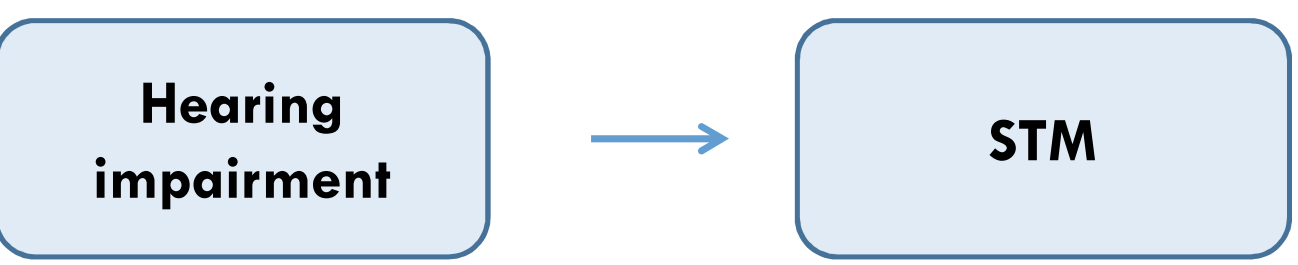

Rabbit (1991) : old participants with a mild hearing loss recalled fewer words than old participants with a good hearing

How could hearing impairment interfere with STM capacities?

$\square$ The effortfulness hypothesis (Rabbit 1968; 1991)

\section{Attentional resources}

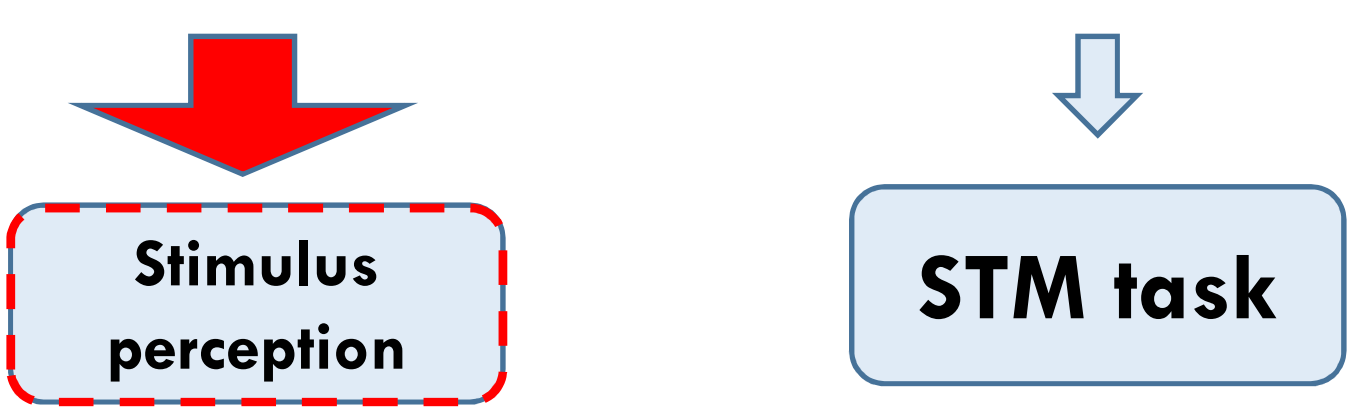




\section{Hypotheses}

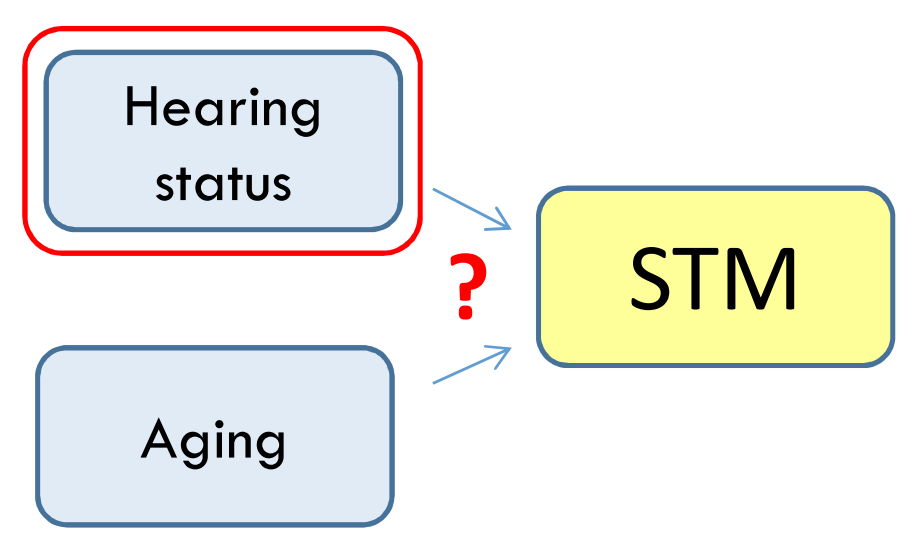

Older participants with a hearing loss

$>$ Young participants with a hearing loss, matched for hearing thresholds

$>$ Young participants with no hearing loss 
METHODS 


\section{Participants}

\begin{tabular}{|c|c|c|c|}
\hline Variable & Controls & $\begin{array}{l}\text { Young hearing- } \\
\text { matched }\end{array}$ & Elderly \\
\hline Number of participants & 16 & 16 & 16 \\
\hline Age (years) & $18-30$ & $18-30$ & $60-75$ \\
\hline Gender (M/F) & $6 / 10$ & $5 / 11$ & $5 / 11$ \\
\hline Hearing threshold (dB HL) & $7.12(2.16)$ & $17.20(5.34)$ & $18.76(5.56)$ \\
\hline Vocabulary level & $0.77(0.09)$ & $0.77(0.08)$ & $0.86(0.09)$ \\
\hline \multicolumn{4}{|l|}{ Mill Hill (proportions) } \\
\hline Processing speed (ms). & 395.64180 & $398.47(62.14)$ & $475.410(110.94)$ \\
\hline
\end{tabular}




\section{Participants}

$\square$ Matched for academic background : $\geq 12$ years of schooling

$\square$ Native French speakers

$\square$ Corrected or normal vision

$\square$ No hearing aid

$\square$ No

口 dementia (Mattis Scale > 130/144)

$\square$ neurological, neuropschological, psychiatric disorders

$\square$ medication use 


\section{Materials:}

口 STM memory tasks

$\square \quad$ Immediate serial recall of high frequency words

- Immediate serial recall of similar and dissimilar words

$\square$ Serial order reconstruction task

$\square \quad$ Online phonological processing

口 Speeded nonword repetition task 


\section{Materials: Immediate Serial Recall (ISR)}

$\square \quad$ ISR of high frequency words (Majerus, 2006)

$>$ High frequency CVC words (Content, Mousty, \& Radeau, 1990)

$>$ Lists with increasing length (1 to 6 items)

"Sauce, balle, taupe "

"Sauce, balle, taupe"

$\%$ words correctly recalled in their order of presentation

¿Phonological processing

$>$ Words not known in advance

$>$ Open pool

$>$ New on every trial 


\section{Materials: Immediate Serial Recall}

- ISR of similar and dissimilar words (Majerus, 2005)

$>8$ similar and 8 dissimilar words of moderate frequency (Content et al., 1990)

$>$ Matched for imageability

$>$ Lists with increasing length (2 to 8 items)

$>$ Similar words: differed only by the onset phoneme (e.g., bois-roi)

"roi, loi, bois"

(1) "roi, loi, bois"

$>\%$ words correctly recalled in their order of presentation

Similar condition : $\Uparrow$ Phonological processing : almost identical words 


\section{Materials: serial order reconstruction}

Serial order reconstruction task (Majerus, 2005)

$>$ Digits from 1 to 9

$>$ Lists with increasing length (3 to 9)

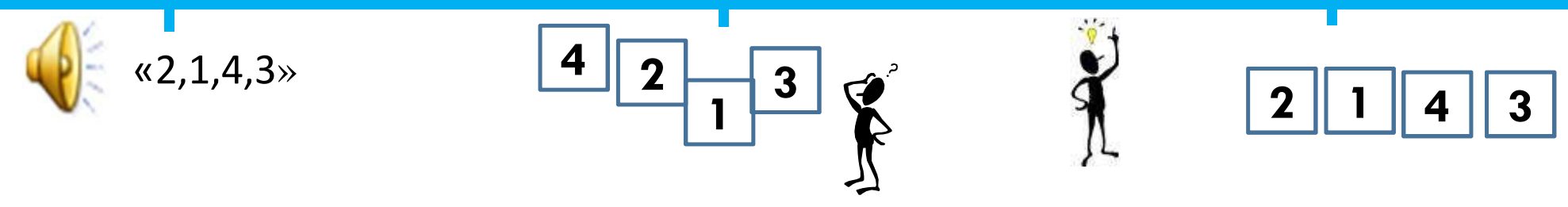

$>$ \% digits correctly replaced in their order of presentation

Focused on the order of the items presented

R Phonological processing

$>$ Highly familiar stimuli

$>$ Known in advance

$>$ Provided at recall (cards representing digits were given) 


\section{Materials: phonological processing}

$\square$ Speeded nonword repetition task

口 Impaired performance in STM $\rightarrow$ items mispercieved?

$\square$ Online phonological processing of auditory items

口 Minimal STM load

$\square$ Nonwords presented in isolation

Task $=$ recall the nonwords

口 CVC - low phonotactic frequency (Tubach \& Boë, 1990) 


\section{RESULTS}




\section{ISR of high frequency words}

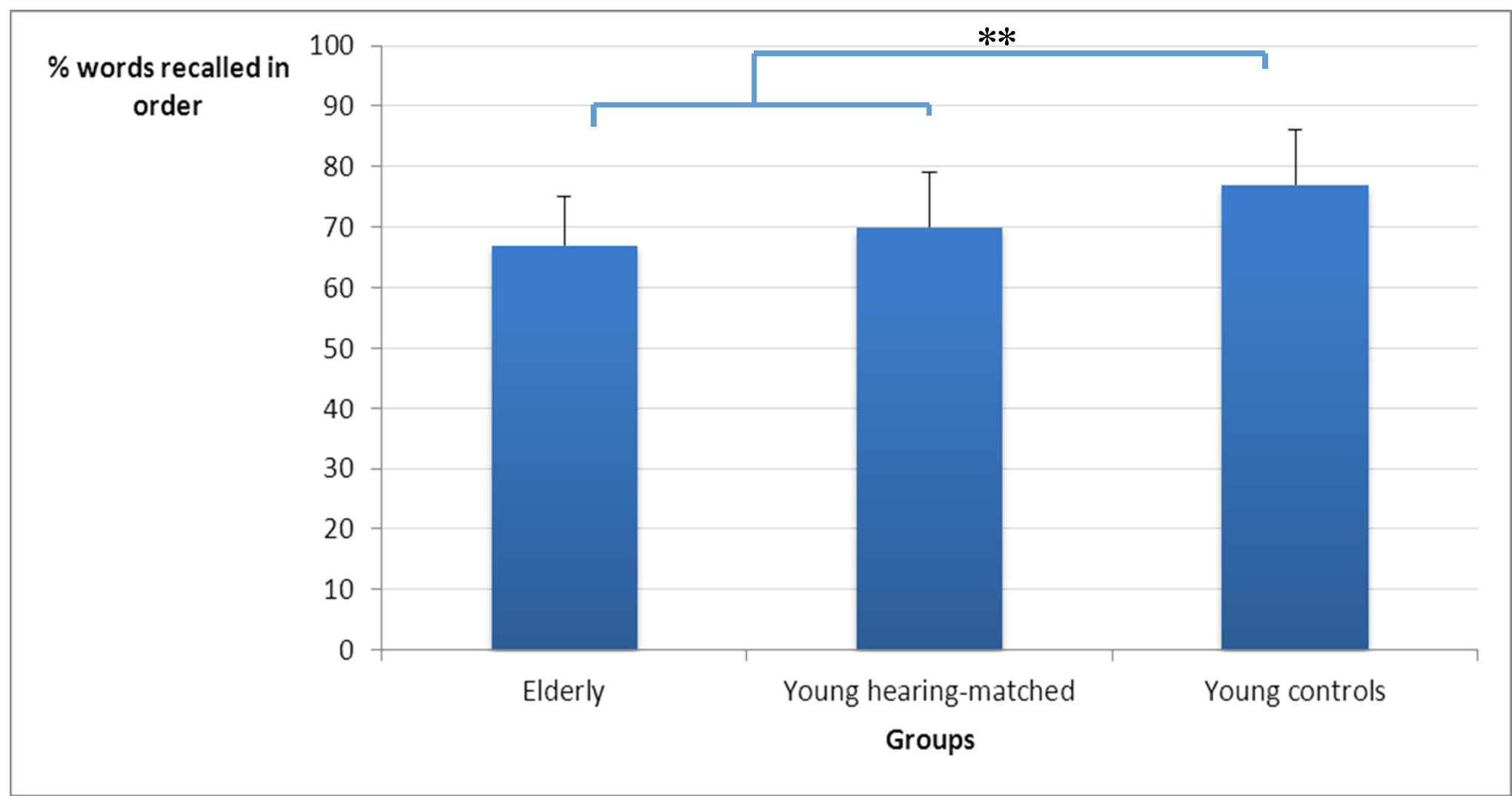

$$
F(2,45)=5.60, p<.01, \quad=.19
$$




\section{ISR of phonologically similar and dissimilar words}

Similar condition

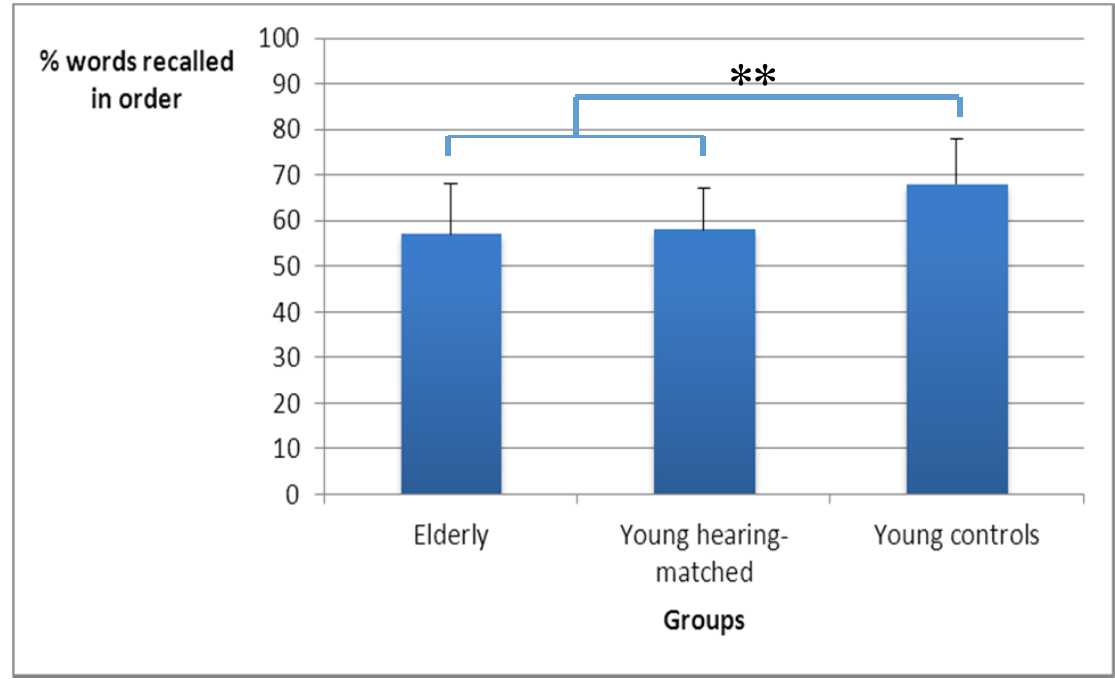

$F(2,45)=6.89, p<.01, \quad=.23$

\section{Dissimilar condition}

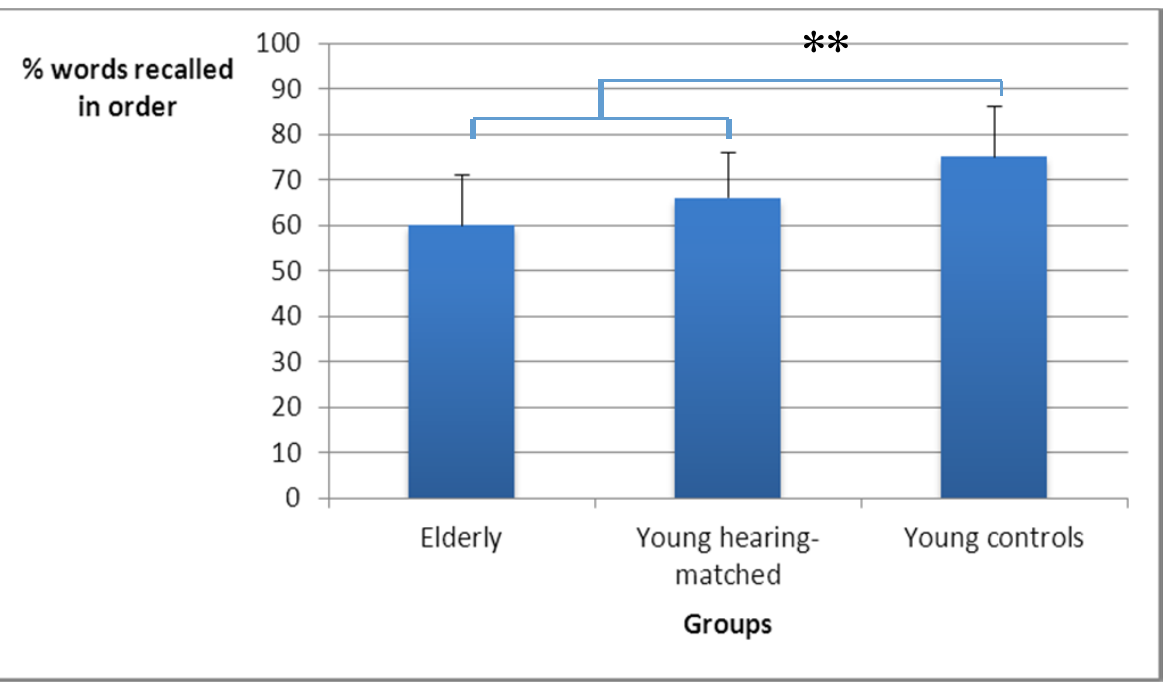

$$
F(2,45)=7.99, p<.01, \quad=.26
$$




\section{Serial order reconstruction task}

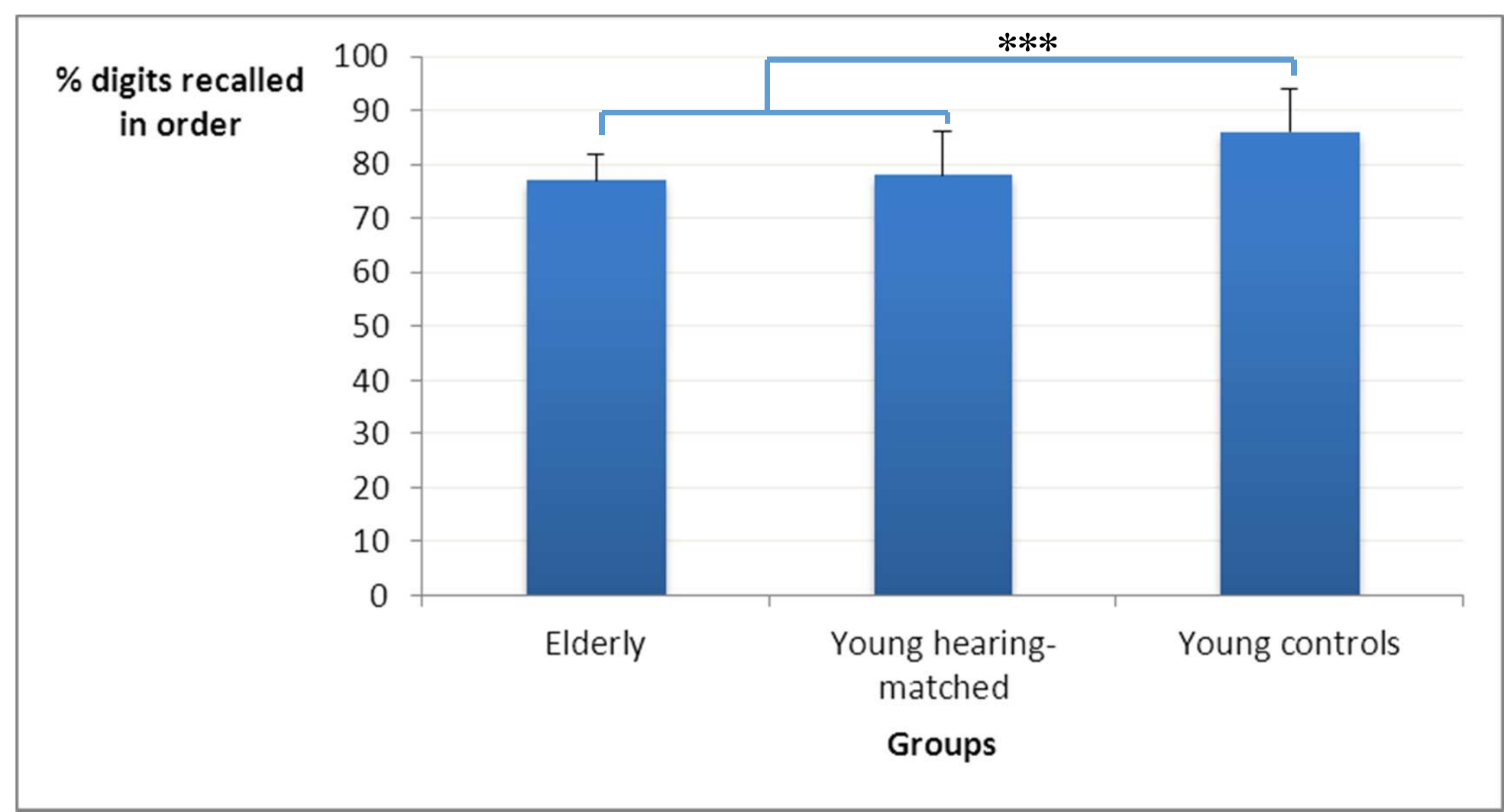

$F(2,45)=7.55, p<.001 ; \quad=.25$ 


\section{Speeded nonword repetition task}

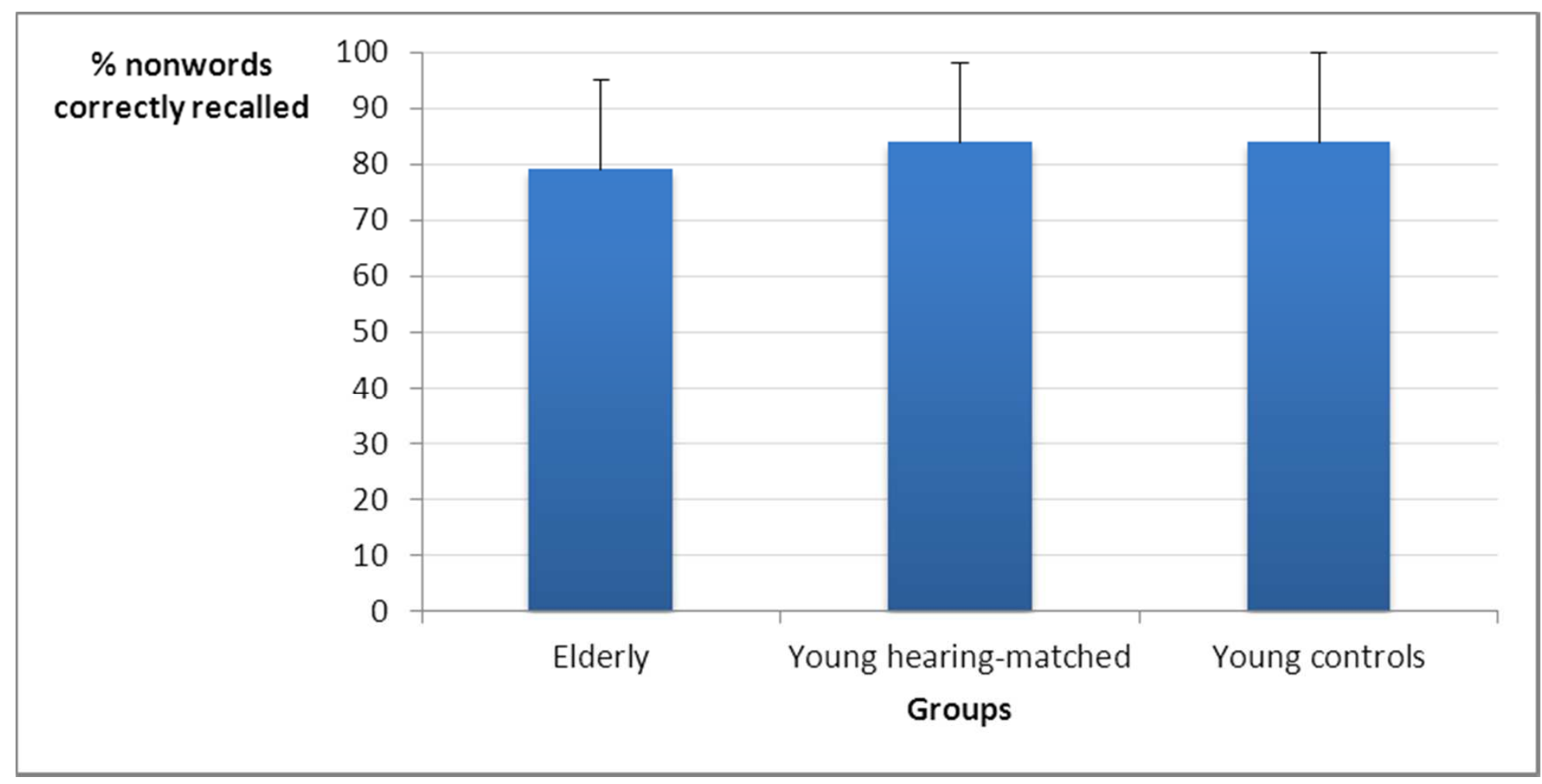

$F(2,45)=.48$, ns. 


\section{DISCUSSION}




\section{Hearing loss}

\section{STM}

> Speeded nonword repetition task: items well percieved

$>$ In STM tasks, performance decreased. Why?

$\square \quad$ The effortfulness hypothesis (Rabbit 1968; 1991)

\section{Attentional resources}

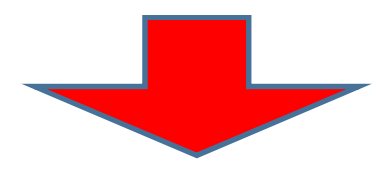

Stimulus perception
STM task 


\section{STM for serial order and STM for item information}

$\square$ In typical STM tasks (e.g., digit span tasks), simultaneous storage of:

$\checkmark$ Item information: phonological and semantic properties

$\checkmark$ Order information: sequential order of the items

$\square$ Different mechanisms for the storage of item and order information

- Experimental studies (e.g., Saint-Aubin \& Poirier, 1999; Majerus et al., 2006b; Nairne \& Kelly, 2004)

- Neuropsychological data (e.g., Brock et al., 2004; Majerus et al. 2006a, 2007b)

- Neuroimaging data (e.g., Majerus et al., 2006c, 2007a) 
Majerus (2008); Majerus, Heiligenstein, Gautherot, Poncelet, and Van der Linden (2009); Majerus (2010)

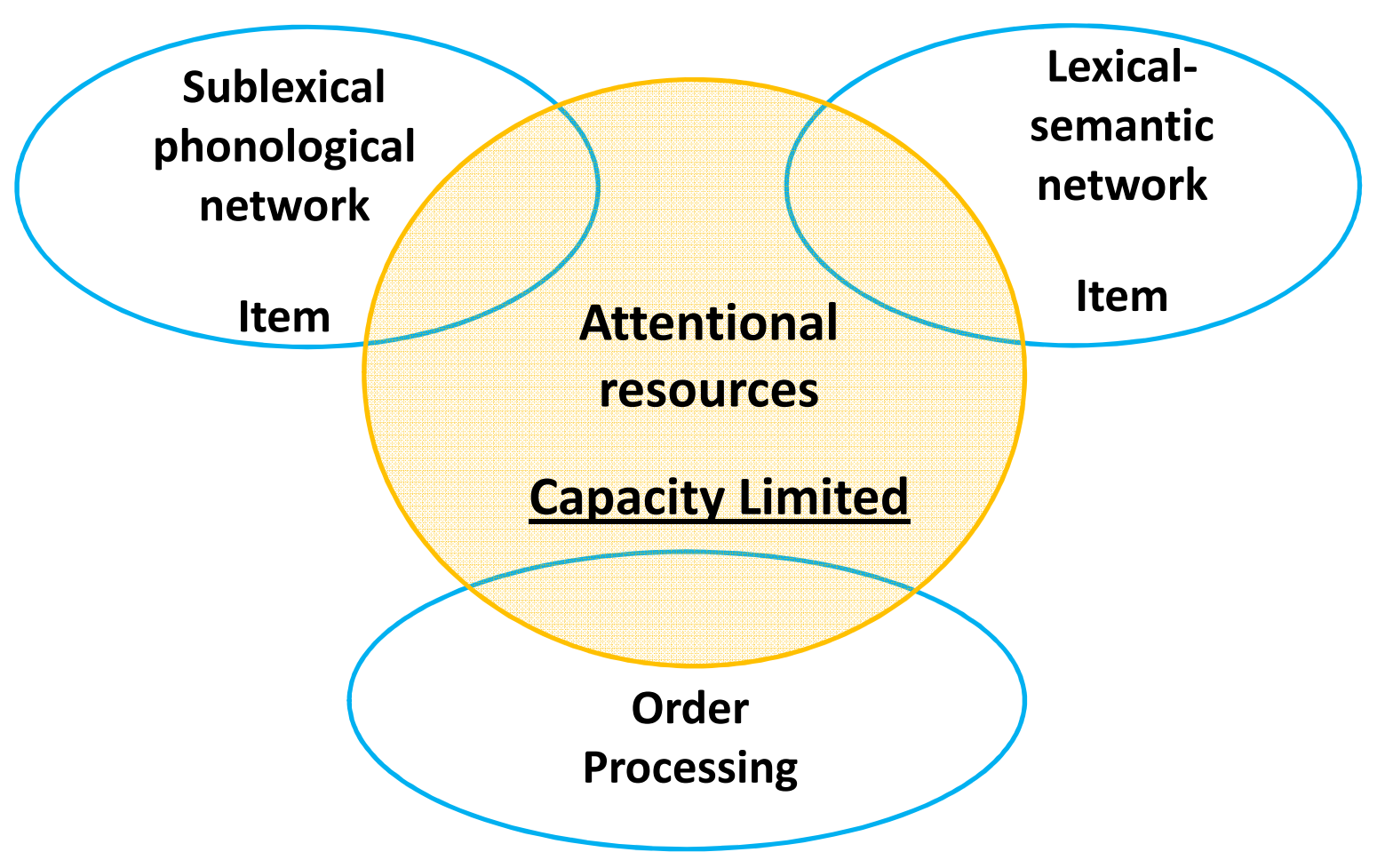




\section{Conclusions...}

$\square$ Auditory factor influences STM more than does aging

$\square$ Auditory impairment $\rightarrow$ Attentional resources that would be otherwise available for STM 


\section{THANK YOU FOR YOUR ATTENTION}

